\title{
Dense granular Flows: a conceptual design of high-power neutron source
}

\author{
Lei Yang ${ }^{1, ~ *}$, Sheng Zhang ${ }^{1}$, Ping Lin ${ }^{1}$, Guanghui Yang ${ }^{1,2}$, Yuan Tian ${ }^{1}$, Jiang-feng Wan ${ }^{1,2}$ \\ ${ }^{1}$ Institute of Modern Physics, Chinese Academy of Sciences, 730000, Nanchang Road 509, Chengguan, Lanzhou, China \\ ${ }^{2}$ Univeristy of Chinese Academy of Sciences, 100049, Yuquan Road 19 A, Shijingshan, Beijing, China
}

\begin{abstract}
A high-power neutron source system is very useful for multifunctional applications, such as material facilities for advanced nuclear power, space radiation studies, radiography and tomography. Here the idea of inclined dense granular flow is utilized and developed in a new conceptual design of a compact high power target to produce a high-energy and high-flux neutron irradiation (the flux is up to $10^{15} \mathrm{n} / \mathrm{cm}^{2} / \mathrm{s}$ or even $10^{16}$ ). Comparing to the traditional solid and liquid heavy metal targets, this design has advantages in material choice, fluid stability, heat removal, etc. In this paper the natures of the granular flows in an inclined chute are investigated and preliminary experimental and numerical results are reported. Then the feasibility of this design is discussed.
\end{abstract}

\section{Background}

In many areas of physics, chemistry, biology, materials, and nuclear engineering, to study the structure and functionality of materials, it is very valuable for a very intense source of neutrons. In complement to X-ray or synchrotron sources, neutron source has several unique advantages [1]. Spallation neutron source is an accelerator-driven facility to produce high intensity neutrons based on spallation reactions. After the successful first spallation neutron sources, several highpower neutron sources are built, constructed and planned, such as SNS [2], J-PARC [3] and ESS [4].

For engineering of spallation target, there are many design options such as plate targets, rod targets, heavy metal targets or rotation targets [5]. Recently, the new concept of gravity-driven Dense Granular Target (DGT) was proposed [6]. This new concept will contribute to the design of MW spallation target for CIADS project [7] (see details in Figure 1).

Compared to currently wide used targets, the attractions for DGT include:

1) The flowing behaviors of grains in DGT are analogous to the fluids [8] and the deposited high power will be removed off-line.

2) In DGT, heat shocks induced by proton beam are dispersed since granular materials usually show an excellent buffering performance [9].

3) Grains in DGT are renewed continuously off line. There are benefits in selecting proper materials to reduce the corrosion, chemistry-toxicity and radio-toxicity.

4) There are mature technologies in industry capable for conveying, filtering and cooling grains.

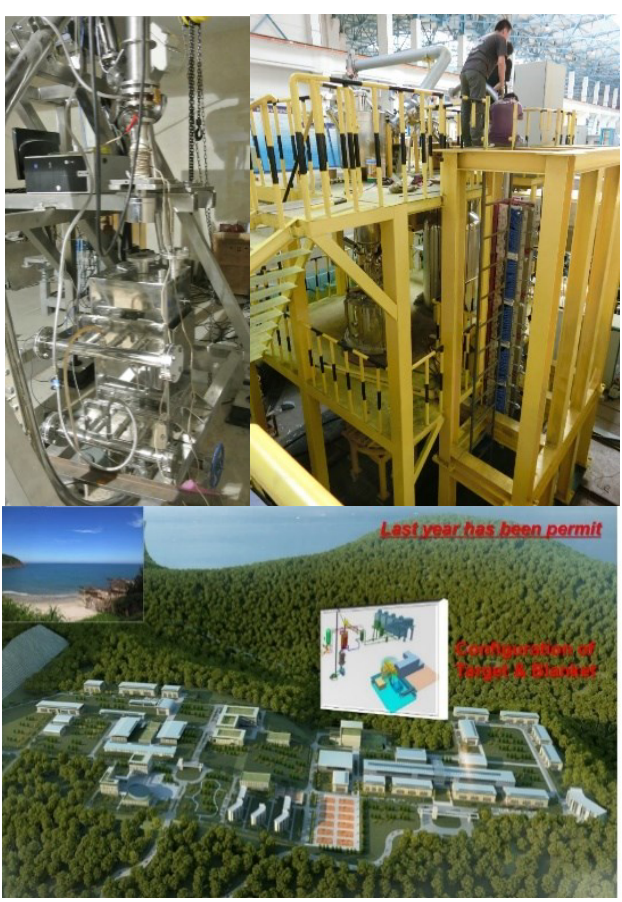

Fig. 1. Engineering application of granular target was put into practice during the target research of ADS project, which is supported by the "Strategic Priority Research Program" of the Chinese Academy of Sciences. Top-left: A principle testing facility coupled with $1.2 \mathrm{MeV}$ electron beam was operated in 2013. Top-right: Later in 2015, a large scale facility started its construction. Bottom: Sup ported by these preliminary work, the next stage namely CIADS project will setup a prototype of ADS system applying granular target, in Huizhou, Guangdong.

For the nuclear and space engineering, a strong and rapid testing facility is much needed. Here a conceptual design of a high-energy and high-flux neutron source is

\footnotetext{
* Corresponding author: lyang@impcas.ac.cn
}

(C) The Authors, published by EDP Sciences. This is an open access article distributed under the terms of the Creative Commons Attribution License 4.0 (http://creativecommons.org/licenses/by/4.0/). 
proposed which is able to offer a neutron flux up to $10^{15}$ $\mathrm{n} / \mathrm{cm}^{2} / \mathrm{s}$. Due to a hopper was used as the container of DGT in [6], the placement of specimens is limited. For the new inclined granular flow target, the flow can be easily controlled and modified [10], which will be shown below. The dynamics and heat transfer in this target are plotted after a series of large-scale simulations.

Table 1. Geometrical and material parameters in this paper.

\begin{tabular}{|c|c|c|}
\hline Quantity(Units) & Symbol & Value \\
\hline Diameter of particles $(\mathrm{mm})$ & $d$ & 5 \\
\hline Width of the chute $(d)$ & $W$ & 40 \\
\hline Length of the chute $(d)$ & $L$ & 600 \\
\hline Height of the gate $(d)$ & $G$ & 25 \\
\hline $\begin{array}{l}\text { Inclination angle of the } \\
\text { chute }\left({ }^{\circ}\right)\end{array}$ & $\theta_{c}$ & 25 \\
\hline $\begin{array}{l}\text { Length/width of the } \\
\text { reservoir }(d)\end{array}$ & $D$ & 40 \\
\hline Elastic modulus (GPa) & E & 287 \\
\hline Poisson's ratio & $v$ & 0.032 \\
\hline $\begin{array}{l}\text { Particle-particle and } \\
\text { particle-wall friction } \\
\text { coefficients }\end{array}$ & $\mu$ & 0.2 \\
\hline Density $\left(\mathrm{kg} / \mathrm{m}^{3}\right)$ & $\rho$ & 1850 \\
\hline $\begin{array}{l}\text { Particle-particle and } \\
\text { particle-wall coefficient of } \\
\text { restitution }\end{array}$ & $\varepsilon$ & 0.9 \\
\hline $\begin{array}{l}\text { Thermal conductivity of } \\
\text { particles }(\mathrm{W} / \mathrm{m} / \mathrm{K})\end{array}$ & $\lambda_{p}$ & 200 \\
\hline $\begin{array}{l}\text { Coefficient of linear } \\
\text { thermal expansion }\left(10^{-6} / \mathrm{K}\right)\end{array}$ & $\alpha$ & 11.3 \\
\hline Heat capacity $(\mathrm{J} / \mathrm{K} / \mathrm{kg})$ & $C_{p}$ & 1825 \\
\hline Hardness (GPa) & $H$ & 1.67 \\
\hline $\begin{array}{l}\text { Thermal conductivity of } \\
\text { gas }(\mathrm{W} / \mathrm{m} / \mathrm{K})\end{array}$ & $\lambda_{g}$ & 0.1517 \\
\hline
\end{tabular}

\section{Flow behaviors}

DEM (Discrete Element Method), closely related to MD (Molecular Dynamics), is a widely-accepted method for simulating contact dynamics in many-body systems consisting of particles, such as powders, bubbles, grains and colloids. To simulate the granular flows in the proposed target, a DEM code containing heat-transfer module was run on GPUs (Graphic Processing Units) [1114].

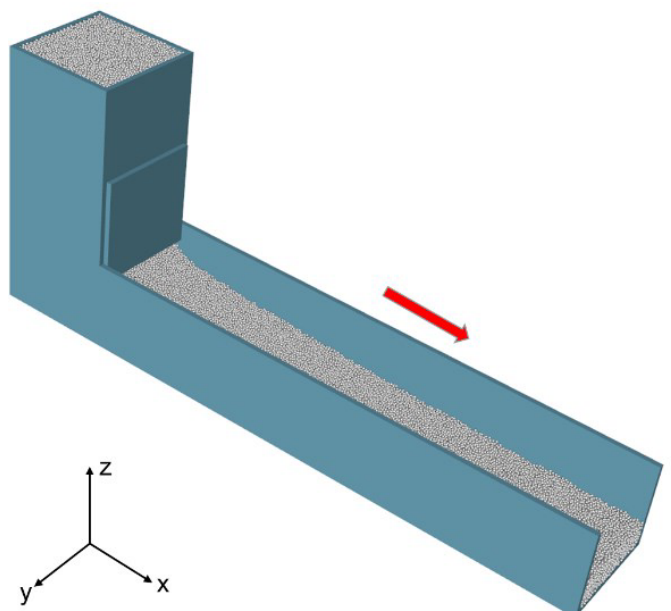

Fig. 2. Configuration of the simple model of chute flows. The red arrow denotes the flowing direction.
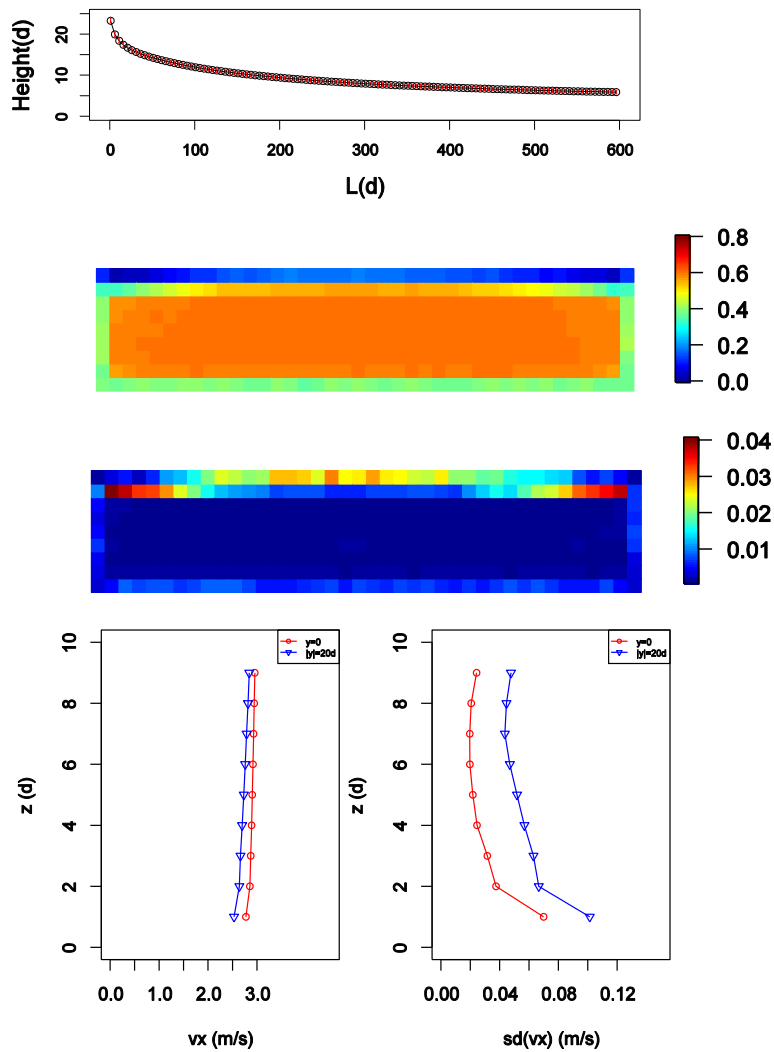

Fig. 3. The simulation results of granular flows in a chute with gate height of $25 d$, chute width of $40 d$ and inclination angle of $25^{\circ}$. Top: the variation of the shape of the flow. Middle-top: spatial profile of volume fraction in the cross section which is perpendicular to $x$ direction at $x=1500 \mathrm{~mm}$. Middle-bottom: Spatial profile of standard deviation of volume fraction in the cross section which is perpendicular to $x$ direction at $x=1,500$ $\mathrm{mm}$. Bottom-left: spatial profile of $v_{x}$ with different depths at $x=1,500 \mathrm{~mm}$. Bottom-right: spatial profile of standard deviation of $v_{x}$ with different depths at $x=1,500 \mathrm{~mm}$. Red circles represent $v_{x}$ of the central particles and blue inverse triangles represent $v_{x}$ of the particles near the sidewalls. 
To simplify the model, monosized beryllium particles (diameter $d$ is $5 \mathrm{~mm}$ ) are simulated. The material of the reservoir and chute is the same as particles and their roughness is assumed as nil. The configuration of the 'the basic' case is schematically shown in Figure 2. The geometrical and material parameters in the basic case are shown in Table 1. The inclination angle is set as $25^{\circ}$ to avoid potential stagnant or gaseous state in the downstream flow [15]. Initially the particles are packed in the reservoir and then are discharged through an opening gate. The time-averaged velocity and volume fraction fields along the chute flow are plotted and the stability of the flow is analyzed.

For this design, the stability of the flow is very important. The morphology of the flow is stable over time (see in Figure 3). To evaluate the stability of the flow, temporal profiles of velocity and volume fraction fields are analyzed and their standard error is calculated. Here it is showed that the standard errors of volume fraction are very small except at the free surface. For $v_{x}$, the standard error on the bottom is more than the standard error at the free surface and although both are relatively small.

\section{Neutronics study}

The results of deuterium beams hitting a monolithic beryllium target are calculated by using GMT (GPU based Monte Carlo Transport program) [16] and checked by Geant4 [17]. The power density distributions along beam direction for different beam energies are plotted in Figure 4 . When the beam energy rises from $10 \mathrm{MeV}$ to 80 $\mathrm{MeV}$, the maximum penetration depth grows from $0.1 \mathrm{~cm}$ to $2.0 \mathrm{~cm}$ and the maximum power density in the target decreases slightly. This profile is used for the following simulations of heat transfer.

A beryllium target bombarded by an $80 \mathrm{MeV}, 20 \mathrm{~mA}$ deuterium beam is simulated and the energy spectrum of neutrons is shown in Figure 5. It is showed that this target will provide sufficient high-energy neutrons to replicate the radiation environment inside fission and fusion reactors. The higher energy neutrons can be produced to satisfy the DEMO $14 \mathrm{MeV}$ peak by increasing the energy of the beam. On the other side, the low-energy neutrons can be produced by utilizing moderators.

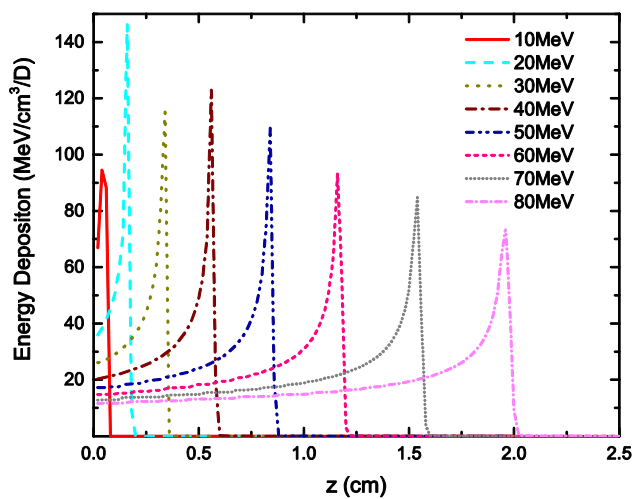

Fig. 4. The profiles of power density distributions along beam direction with different energy deuterium beams.

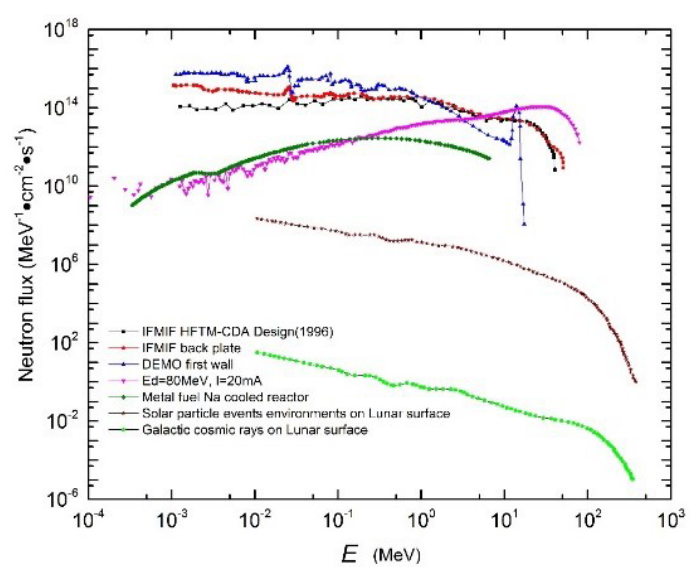

Fig. 5. The energy spectrum of neutrons in different situations. Pink inverse triangles denote the spectrum in the current design.
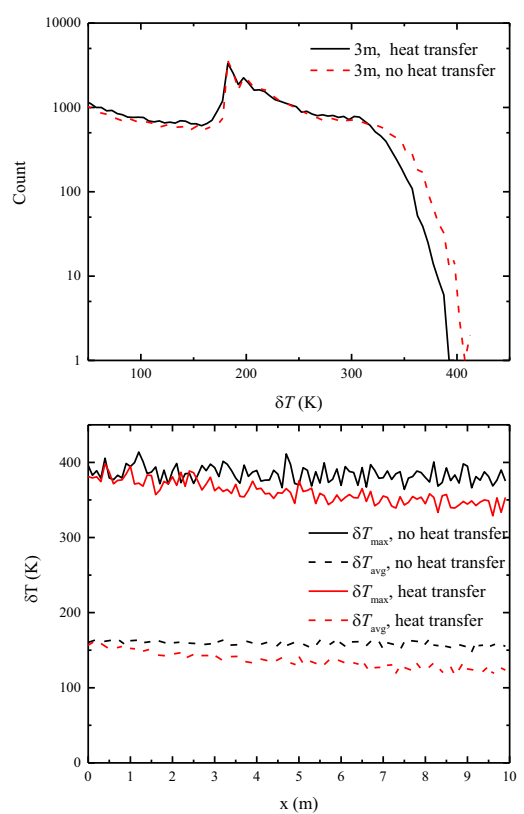

Fig. 6. Top: the distribution of temperature rises. Bottom: the distribution of temperature profile along the $x$ direction.

\section{Heat limit}

The heat transfer process in chute flow with an $80 \mathrm{MeV}$, $20 \mathrm{~mA}$ deuterium beam (beam spot size is $20 \times 20 \mathrm{~mm}^{2}$ ) is simulated. It is supposed that the system is operated in a Helium environment ( $1 \mathrm{~atm})$. The distances between release gate and the shooting point $L$ varies between 2,000 and $3,000 \mathrm{~mm}$. The profile of energy deposition (shown in Figure 4) is used here. In this simulation, four modes of heat transfer are considered: a) thermal conduction through the particle contacts, b) thermal conduction through the stagnant gas film, c) thermal conduction through the contact gaps between two particles, d) radiant heat transfer between surface of particles [18]. The material parameters used for the heat transfer models are listed in Table 1. In this simulation the temperature of particles released from reservoir is set around $290{ }^{\circ} \mathrm{C}$. Quantitative results are shown in Figure 6 (where $L=$ $3,000 \mathrm{~mm}$ ). For the case $L=3,000 \mathrm{~mm}$, the ratio of 
particles whose temperature rise is more than $400{ }^{\circ} \mathrm{C}$ is small (less than 5\%). The average temperature rise of particles is less than $180^{\circ} \mathrm{C}$ and the maximum temperature rise is no more than $400{ }^{\circ} \mathrm{C}$. For comparison, a system without heat transfer is also simulated, where each particle is assumed to be thermally insulated. In this system, the maximum temperature rise is no more than $420^{\circ} \mathrm{C}$ (can be used as a maximum estimate of maximum temperature rise).

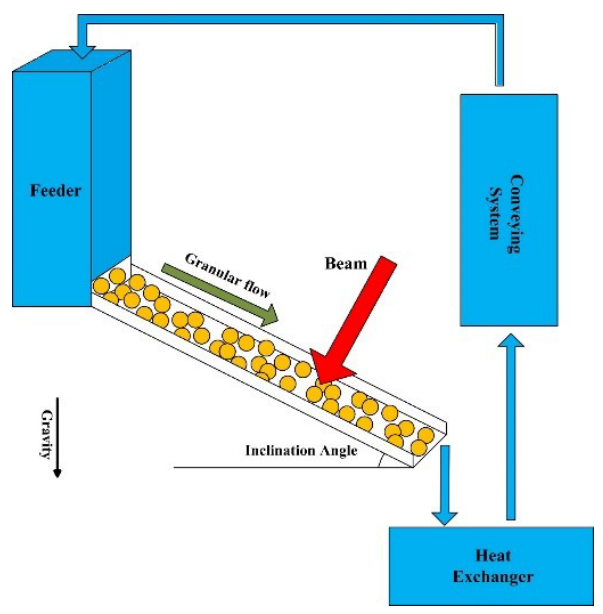

Fig. 7. Schematic diagram of the gravity-driven chute flow target system.

\section{Conceptual design and Discussions}

A conceptual design of the gravity-driven chute flow target is schematically shown in Figure 7. As a feeder, there is a reservoir or a hopper filled with heavy metal particles. Downstream there is an inclined chute and the particles discharging from the feeder will flow down the chute by gravity. The beam hits the flowing particles at a fixed position. At the end of the chute, the particles will flow into a heat exchanger where they are cooled. After filtering, the particles are re-injected into the feeder by a conveying system. The stability of the flows is illustrated above and can be conveniently controlled by adjusting the inclination angles of chute. The chute can be polished to avoid the surface waves mentioned above. The numerical study shows that the neutron flux can be up to $5 \times 10^{15}$ $\mathrm{n} / \mathrm{cm}^{2} / \mathrm{s}$ and the temperature rise in the material is acceptable.

This work is supported by the National Magnetic Confinement Fusion Science Program of China (Grant No. 2014GB104002).

\section{References}

1. Wei, J., et al., China Spallation Neutron Source - an overview of application prospects. Chinese Physics C, 2009. 33(11): p. 1033-1042.

2. Gabriel, T.A., J.R. Haines, and T.J. McManamy, Overview of the Spallation Neutron Source (SNS) with emphasis on target systems. Journal of Nuclear Materials, 2003. 318: p. 1-13.
3. Oyama, Y., J-PARC and new era of science. Nuclear Instruments \& Methods in Physics Research Section a-Accelerators Spectrometers Detectors and Associated Equipment, 2006. 562(2): p. 548-552.

4. Lindroos, M., et al., The European Spallation Source. Nuclear Instruments \& Methods in Physics Research Section B-Beam Interactions with Materials and Atoms, 2011. 269(24): p. 3258-3260.

5. Bauer, G.S., Overview on spallation target design concepts and related materials issues. Journal of Nuclear Materials, 2010. 398(1-3): p. 19-27.

6. Yang, L. and W. Zhan, New concept for ADS spallation target: Gravity-driven dense granular flow target. Science China Technological Sciences, 2015: p. 1-7.

7. Zhan, W. ADS Programme and Key Technology R\&D in China. in Proc. 2013 International Particle Accelerator Conference, Shanghai. 2013.

8. Massoudi, M. and T.X. Phuoc, Conduction and dissipation in the shearing flow of granular materials modeled as non-Newtonian fluids. Powder Technology, 2007. 175(3): p. 146-162.

9. Grujicic, M., et al., Shock-Wave Attenuation and Energy-Dissipation Potential of Granular Materials. Journal of Materials Engineering and Performance, 2012. 21(2): p. 167-179.

10. Silbert, L.E., et al., Granular flow down an inclined plane: Bagnold scaling and rheology. Physical Review E, 2001. 64(5).

11. Tian, Y., et al. A heterogeneous CPU-GPU implementation for discrete elements simulation with multiple GPUs. in Awareness Science and Technology and Ubi-Media Computing (iCAST UMEDIA), 2013 International Joint Conference on. 2013. IEEE.

12. Qi, J., et al., GPU-accelerated DEM implementation with CUDA. International Journal of Computer Science and Engineering, Inderscience, 2015. 11(3): p. 330-337.

13. Zhang, S., et al., Investigating the influence of wall frictions on hopper flows. Granular Matter, 2014. 16(6): p. 857-866.

14. Lin, P., et al., Numerical study of free-fall arches in hopper flows. Physica a-Statistical Mechanics and Its Applications, 2015. 417: p. 29-40.

15. Borzs onyi, T. and R.E. Ecke, Rapid granular flows on a rough incline: Phase diagram, gas transition, and effects of air drag. Physical Review E, 2006. 74(6).

16. Cai, H., et al., Code development and target station design study for Chinese Accelerator-Driven System Project. Nuclear Science and Engineering, accept.

17. Agostinelli, S., et al., GEANT4-a simulation toolkit. Nuclear Instruments \& Methods in Physics Res earch Section a-Accelerators Spectrometers Detectors and Associated Equipment, 2003. 506(3): p. 250-303.

18. Vargas-Escobar, W.L., Discrete modelling of heat conduction in granular media. 2002, University of Pitts burgh. 